\title{
Nonlinear interference in crystal superlattices
}

\author{
Anna V. Paterova $\mathbb{D}^{1}$ and Leonid A. Krivitsky (iD ${ }^{1}$
}

\begin{abstract}
Nonlinear interferometers with correlated photons hold promise to advance optical characterization and metrology techniques by improving their performance and affordability. These interferometers offer subshot noise phase sensitivity and enable measurements in detection-challenging regions using inexpensive and efficient components. The sensitivity of nonlinear interferometers, defined by the ability to measure small shifts of interference fringes, can be significantly enhanced by using multiple nonlinear elements, or crystal superlattices. However, to date, experiments with more than two nonlinear elements have not been realized, thus hindering the potential of nonlinear interferometers. Here, we build a nonlinear interferometer with up to five nonlinear elements, referred to as superlattices, in a highly stable and versatile configuration. We study the modification of the interference pattern for different configurations of the superlattices and perform a proof-of-concept gas sensing experiment with enhanced sensitivity. Our approach offers a viable path towards broader adoption of nonlinear interferometers with correlated photons for imaging, interferometry, and spectroscopy.
\end{abstract}

\section{Introduction}

Optical characterization and metrology techniques benefit from using correlated photons, particularly in studies of light-sensitive and fragile biological and chemical samples ${ }^{1,2}$. For example, strong temporal correlations between photons were used for a single-photon calibration of the efficiency of retinal cells ${ }^{3}$ and enhancing the nonlinear response of biological samples ${ }^{4}$. Furthermore, two-photon interference effects have formed the basis for dispersion-free optical coherence tomography ${ }^{5-7}$, microscopy with enhanced phase contrast ${ }^{8,9}$, and noiserobust spectroscopy of nanostructures ${ }^{10}$ to name a few.

Recently, the nonlinear interference of correlated photons has attracted particular interest in the context of infrared (IR) metrology and sensing ${ }^{11-15}$. A nonlinear interferometer is composed of two nonlinear elements, which produce pairs of correlated photons (signal and idler) under coherent excitation. The signal (in the visible range) and idler (in the IR range) photons are mixed in the interferometric setup, and as long as one cannot distinguish which nonlinear element produced the photons, interference fringes are observed. The interference pattern of signal photons depends on the phases and

\footnotetext{
Correspondence: Leonid A. Krivitsky (Leonid_Krivitskiy@imre.a-star.edu.sg) ${ }^{1}$ Institute of Materials Research and Engineering (IMRE), Agency for Science Technology and Research (A*STAR), 138634 Singapore, Singapore
}

amplitudes of the signal, idler, and pump photons. When idler photons interact with a sample, its properties in the IR range can be inferred from the interference pattern of signal photons in the visible range. Thus, this technique addresses practical challenges of generation and detection of IR light since the sample response is obtained using accessible components for visible light.

Nonlinear interferometers have been realized using numerous physical platforms, including bulk nonlinear crystals $^{11-14,16-18}$, gas cells ${ }^{19}$, fiberized networks ${ }^{20,21}$, and nonlinear waveguides ${ }^{22,23}$. Additionally, nonlinear interferometers have been used for imaging ${ }^{24}$, spectroscopy $^{16,25-27}$, optical coherence tomography ${ }^{28,29}$, superresolution interferometry ${ }^{18,19}$, and polarimetry ${ }^{30}$. All these techniques are intrinsically interferometric. Hence, their sensitivity is defined by the ability to detect small changes in the interference pattern, such as a shift of the fringes or change in the fringe visibility.

One possible way to enhance the sensitivity of nonlinear interferometers was outlined by D. Klyshko ${ }^{31}$, who considered a setup with $N$ identical nonlinear elements separated by linear gaps, referred to here as a crystal superlattice. He showed that with an increase in the number of crystals, bright interference fringes in the frequency domain narrow, yet the spacing between fringes remains unchanged. This idea was theoretically expanded 
in more recent works ${ }^{20,32,33}$; however, to the best of our knowledge, there are no reports on the experimental realization of nonlinear interferometers with more than two nonlinear elements. The major challenges in practical realization are associated with (1) the necessity of superimposing signal and idler modes from multiple nonlinear elements while preserving the quantum indistinguishability, and (2) the necessity to align and stabilize increasingly complex setups.

Here, for the first time, we realize a nonlinear interferometer with a crystal superlattice consisting of up to five nonlinear elements. In our setup, nonlinear elements are arranged sequentially and are pumped by a single coherent laser. By careful design and alignment, we achieve a robust mode overlap of signal and idler photons with remarkable stability. We observe the interference pattern in the frequency-angular spectrum with full flexibility of crystal arrangements and theoretically describe this effect. We also perform a proofof-concept gas sensing experiment with enhanced sensitivity.

\section{Results}

\section{Theoretical framework}

Earlier works theoretically analysed the multicrystal interference in the frequency domain for a single spatial mode $^{31,34,35}$. Analysis of the interference in both the frequency and spatial domains was limited to only two nonlinear crystals ${ }^{36,37}$. Here, we analyse the frequencyangular spectrum obtained in a nonlinear interferometer with $N$ crystals. Let us consider $N$ identical nonlinear crystals of length $l$ separated by $N-1$ equal linear gaps $l$; see Fig. 1a. The crystals are pumped by a coherent laser, and each crystal produces signal $(s)$ and idler $(i)$ photons via spontaneous parametric down-conversion (SPDC). The down-converted photons from each crystal are redirected to the next crystal. The state of the two-photon field produced by a single crystal is given by ${ }^{34}$ :

$$
|\psi\rangle=|\mathrm{vac}\rangle+\sum_{n} \sum_{k_{s}, k_{i}} f_{n}\left(\vec{k}_{s}, \vec{k}_{i}\right) a_{k_{\mathrm{ns}}}^{+} a_{k_{\mathrm{ni}}}^{+}|\mathrm{vac}\rangle
$$

where $f_{n}\left(\vec{k}_{s}, \vec{k}_{i}\right)$ is the two-photon field amplitude from the $n$-th crystal $n=[1, N], a_{k_{\mathrm{ns}}}^{+}$and $a_{k_{\mathrm{ni}}}^{+}$are creation operators of photons in the $n$-th crystal with wavevectors $\vec{k}_{s}$ and $\vec{k}_{i}$, respectively, and $|\mathrm{vac}\rangle$ indicates the vacuum state. Here, we assume that the photons are generated in the spontaneous regime and that the initial state of the signal and idler photons in each crystal can be considered a vacuum state ${ }^{11,12,31}$.

Assuming the pump is a monochromatic plane wave and the crystal is thin and uniform, the amplitude of a two-photon field is given by ${ }^{31,38}$ :

$$
f_{n} \propto \chi E_{p} \int_{z_{n}}^{z_{n+l}} d z D_{n}^{* p} D_{n}^{s} D_{n}^{i}
$$

where $\mathrm{X}$ is the second-order susceptibility of the crystal; $z_{n}=-n l+(n-1) l^{\prime}$ is the coordinate of the front edge of the $n$-th nonlinear crystal; $E_{p}$ is the field of the pump; and $D_{n}^{j}$ is the following propagation function for the signal, idler, and pump photons $(j=s, i, p)$ :

$$
D_{n}^{j}\left(k_{j}, z\right)=\exp \left[-i k_{j}^{z} z+(n-1)\left(k_{j}^{\prime z}-k_{j}^{z}\right) l^{\prime}\right]
$$

where $k_{j}^{z}$ and $k_{j}^{\prime z}$ are the longitudinal wavevectors inside the nonlinear crystal and in the gap between crystals, respectively. From Eqs. (2) and (3), we obtain the twophoton field amplitude as follows:

$$
f_{n}=\frac{1-\exp (-i \Delta k l)}{i \Delta k l} \cdot\left[-i(n-1)\left(\Delta k l+\Delta k^{\prime} l^{\prime}\right)\right]
$$

where $\Delta k$ and $\Delta k^{\prime}$ are the wavevector mismatches inside the nonlinear crystal and in the linear gap, respectively. For $N$ identical crystals, the two-photon field amplitude is given by the sum of contributions from individual crystals as follows:

$$
F=\sum_{n=1}^{N} f_{n} \propto \operatorname{sinc}(\Delta k l / 2) \sum_{n=1}^{N} e^{i(n-1) \phi}
$$

where $\phi=\left(\Delta k l+\Delta k^{\prime} l^{\prime}\right)$. Then, from Eq. (5), the intensity distribution of the signal photons as a function of frequency $\omega_{s}$ and scattering angle $\theta_{s}$, as measured in the experiment, is given by ${ }^{31,35,38}$ :

$$
\mathrm{I}_{N}\left(\omega_{s}, \theta_{s}\right)=|F|^{2} \propto\left\{\operatorname{sinc}\left(\frac{\Delta k l}{2}\right) \cdot \frac{\sin [N \phi / 2]}{\sin [\phi / 2]}\right\}^{2}
$$

We express the phase mismatch in the frequency and scattering angle in Section 1 of the Supplementary Materials $^{36,37}$ and plot the interference patterns for the nonlinear interferometer with two and five crystals; see Fig. 2a, b, respectively. Figure 2c shows cross sections of the interference patterns for different numbers of crystals in the superlattice. We see that as the number of crystals increases, the interference maxima become narrower, yet the spacing between them remains unchanged.

In Section 2 of the Supplementary Materials, we show that the width of the bright fringes is inversely 

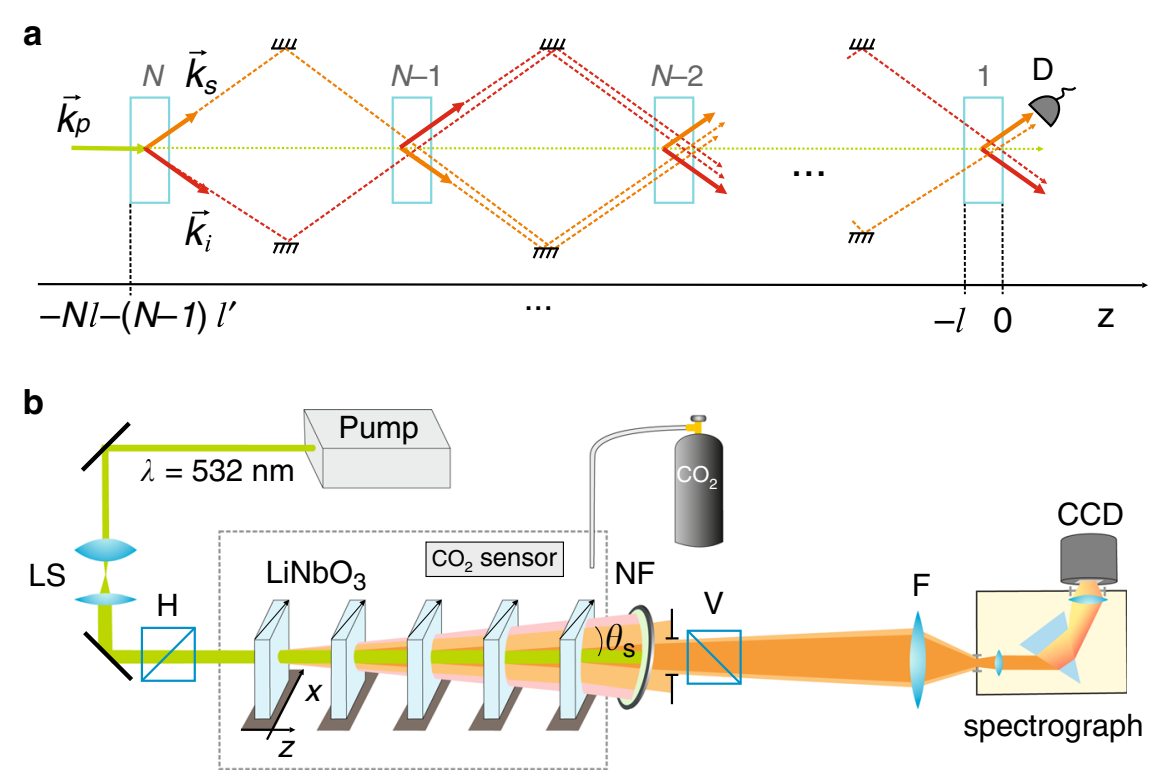

Fig. 1 Nonlinear interferometer with crystal superlattice. a Conceptual scheme. $N$ identical nonlinear crystals, separated by equal gaps, are coherently pumped by a laser (green arrow). In each crystal, the pump photon $k_{p}$ decays into a pair of correlated signal $k_{s}$ (orange arrow) and idler $k_{i}$ (pink arrow) photons, which are then redirected to the next crystal. The intensity of signal photons is measured in the experiments by detector $\mathrm{D}$. The paths of signal and idler photons are disjoined for clarity. $\mathbf{b}$ Experimental realization. A cw laser pumps SPDC crystals. Signal photons (orange) and idler photons (pink) generated in different crystals overlap within the interaction volume defined by the pump. The signal photons are projected by the lens F onto the slit of the imaging spectrograph with the CCD camera. The CCD camera captures the frequency-angular spectra. Optical axes of the crystals are aligned in the same direction (marked by the arrows). In the gas sensing experiments, carbon dioxide gas is injected into the enclosure (marked by a dashed rectangle).
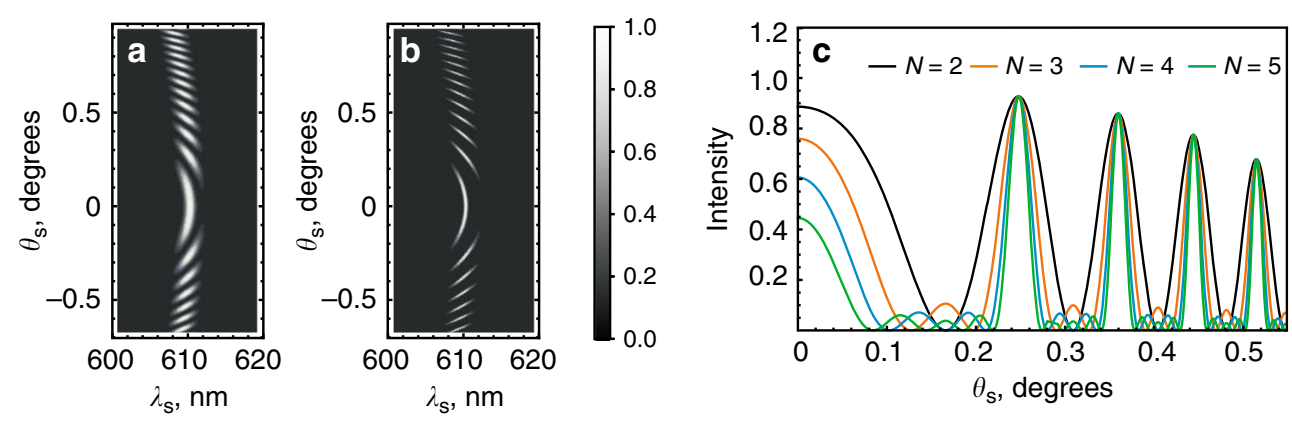

Fig. 2 Theoretical results. Calculated frequency-angular spectra (interference patterns) of signal photons from two $\mathbf{a}$ and five $\mathbf{b}$ identical $\mathrm{LiNbO}_{3}$ crystals (crystal length $I=1 \mathrm{~mm}$, air gaps between crystals $I^{\prime}=8.2 \mathrm{~mm}$, the orientation of the axis $\theta_{c}=50.34^{\circ}$, the pump is a $532 \mathrm{~nm}$ laser). $\mathbf{c}$ Vertical cross sections of $(\mathbf{a})$ and $(\mathbf{b})$ at $\lambda_{s}=610.4 \mathrm{~nm}$ (idler photon wavelength is $\lambda_{i}=4142 \mathrm{~nm}$ ) for different numbers of crystals in the superlattice.

proportional to the number of crystals $N$ :

$$
\delta \theta_{s} \propto \frac{\pi}{N}
$$

From Eq. (7), we note the striking similarity between the interference fringes for the nonlinear interferometer with a crystal superlattice and conventional multi-slit or FabryPerot linear interference ${ }^{39}$.
Observation of the interference with a crystal superlattice

First, we set the phase-matching angle of the crystal to $\theta_{\mathrm{c}}=50.34^{\circ} \pm 0.02^{\circ}$ when the signal SPDC photons are generated at $\sim 610.4 \mathrm{~nm}$ (bandwidth $2 \mathrm{~nm}$ ) and idler photons at $4.14 \mu \mathrm{m}$ (bandwidth $92 \mathrm{~nm}$ ). The normalized frequency-angular spectra of signal photons for two and five nonlinear crystals are shown in Fig. 3a, b, respectively. Our key observation is that the interference fringes for the interferometer with five crystals become narrower than those for the interferometer with two crystals, yet the 


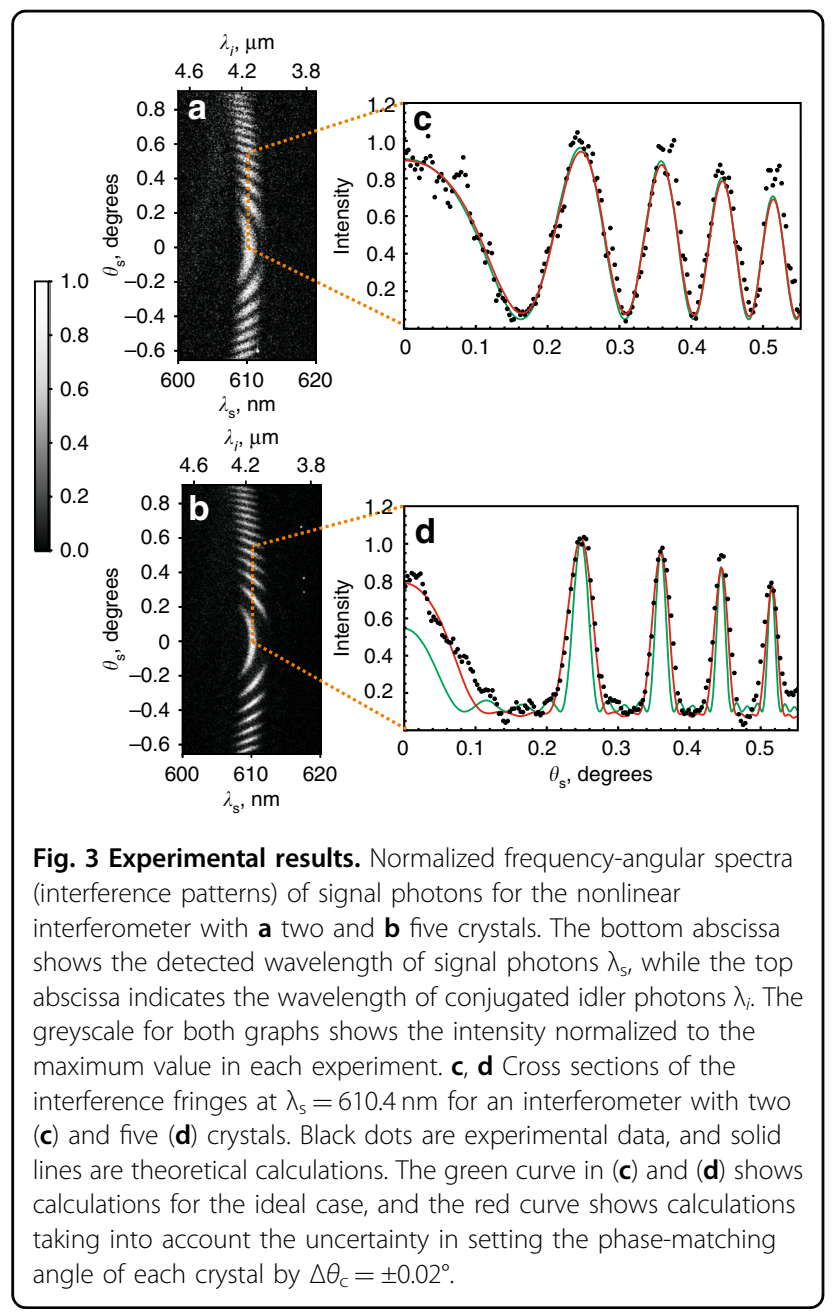

period of the fringes remains unchanged. Figure $3 c, d$ shows the cross sections of the interference pattern at $\lambda_{\mathrm{s}}=610.4 \mathrm{~nm}$ for the interferometer with two and five crystals, respectively. The cross sections are taken by averaging the intensity across the bandwidth of $\Delta \lambda_{s}=$ $0.4 \mathrm{~nm}$. To achieve the same flux of the photons for the two- and five-crystal configurations, the acquisition time is set to 360 and $144 \mathrm{~s}$, respectively.

The solid curves in Fig. 3c, d correspond to theoretical calculations. The green curve shows the theory in the ideal case, and the red curve shows the theory that accounts for the experimental accuracy in setting the phase-matching angle of each crystal to $\Delta \theta_{\mathrm{c}}= \pm 0.02^{\circ}$. We found that a slight misalignment in setting $\theta_{\mathrm{c}}$ becomes crucial for the interferometer with an increasing number of crystals. A detailed analysis of the sensitivity of the interferometer to various experimental parameters is presented in Sections 3 and 4 of the Supplementary Materials.

We experimented with sets of two, three, four, and five crystals in the superlattice. In each case, we fit the experimental data by Eq. (6) and determined the width of

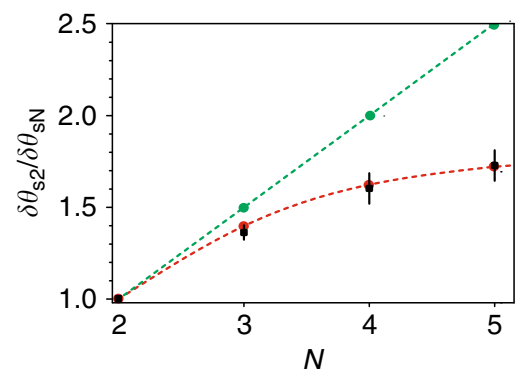

Fig. 4 Scaling of the width of interference fringes. Experimental dependence of the ratio of the width of fringes $\delta \theta_{\mathrm{s} 2} / \delta \theta_{\mathrm{sN}}$ on the number of crystals in the superlattice (black squares). The green dots show theoretical calculations in the ideal case; the red dots show the calculated dependence, which accounts for the experimental uncertainty in setting the phase-matching angle of $\Delta \theta_{c}= \pm 0.02^{\circ}$. Dashed lines are given to guide the eye.

the interference fringes. Figure 4 shows the ratio of the widths of the interference fringes for the $\mathrm{N}$-crystal interferometer $\delta \theta_{s N}$ and two-crystal interferometer $\delta \theta_{s 2}$. The key observation is consistent with the theory: the interference fringes become narrower with increasing number $N$ of nonlinear crystals. The green dots in Fig. 4 show the linear scaling of the relative width in the ideal case; see Eq. (7). The red dots in Fig. 4 show the calculation results taking into account the uncertainty in setting the phasematching angle of each crystal to $\Delta \theta_{\mathrm{c}}= \pm 0.02^{\circ}$, which is consistent with our experimental data, shown by black squares. Note that a stronger dependence on the uncertainty of the experimental parameters in the interferometer with a crystal superlattice is a manifestation of the common property of multielement interferometers.

\section{Proof-of-concept gas sensing experiment}

Next, we set the phase-matching angle to $\theta_{\mathrm{c}}=50.23^{\circ} \pm$ $0.02^{\circ}$ and obtain signal photons at $\lambda_{s}=609.3 \mathrm{~nm}$ and idler photons in the vicinity of the absorption resonance of $\mathrm{CO}_{2}$ at $\lambda_{i}=4.19 \mu \mathrm{m}$. The frequency-angular spectra of signal photons from an interferometer with two and five crystals are shown in Fig. 5a-d, respectively. Figure 5a and c corresponds to the case when there is air in the gap between the crystals, and Fig. $5 \mathrm{~b}$ and d corresponds to the case when $\mathrm{CO}_{2}$ gas is injected in the gaps (concentration $(35 \pm 3.5) \times$ $10^{3} \mathrm{ppm}$ ). Because of the absorption of idler photons by the gas, the interference pattern of signal photons experiences a phase shift and reduction in visibility.

Figure 6 shows the cross sections of the interference pattern at $\lambda_{\mathrm{s}}=609.3 \mathrm{~nm}\left(\lambda_{i}=4.19 \mu \mathrm{m}\right)$ when the wavelength of idler photons is detuned from the absorption resonance of $\mathrm{CO}_{2}$ by $\sim 72 \mathrm{~nm}$. In this case, the gas causes a phase shift of the interference fringes without a significant change in the fringe visibility. Figure $6 \mathrm{a}, \mathrm{b}$ corresponds to the interference fringes for an interferometer with two 


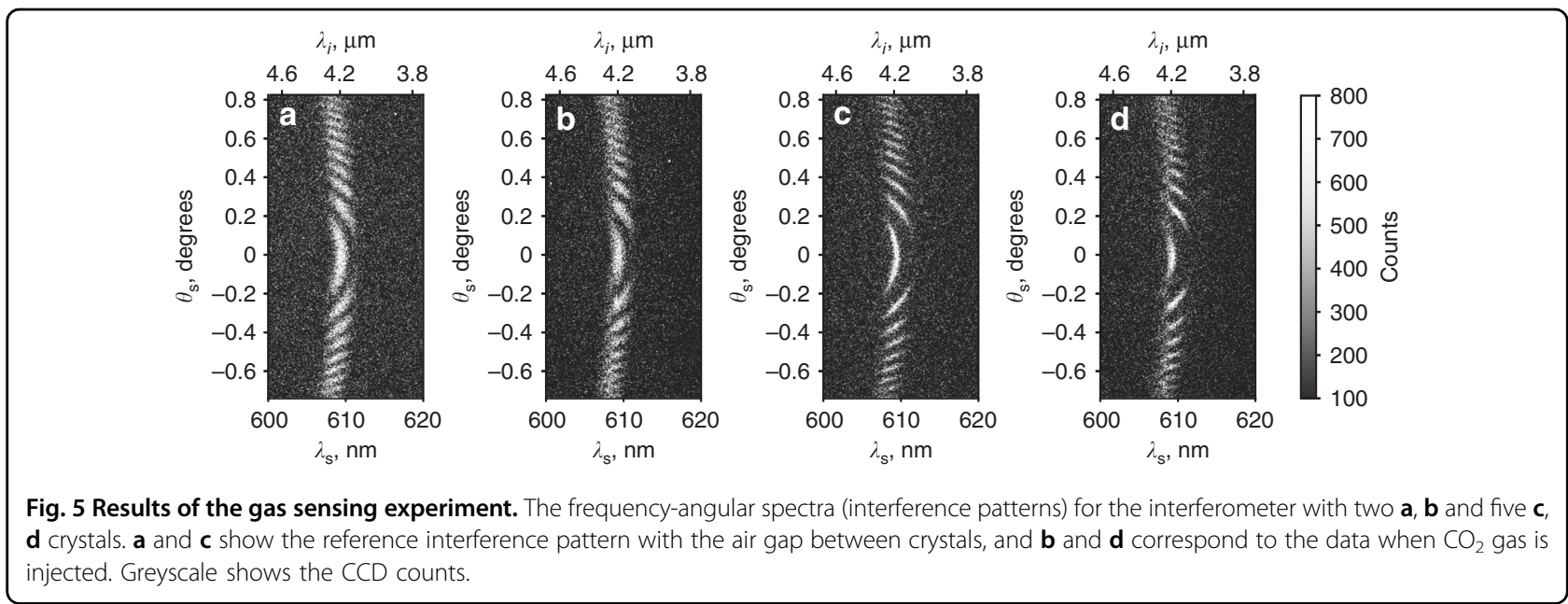
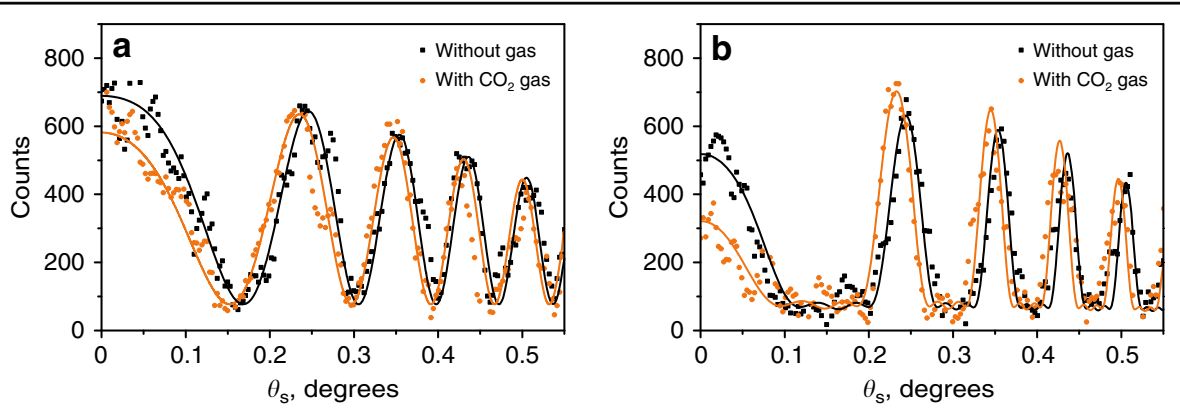

Fig. 6 Analysis of interference fringes for the gas sensing experiment. Cross section of the interference patterns at $\lambda_{\mathrm{s}}=609.3 \mathrm{~nm}$ for a nonlinear interferometer with $\mathbf{a}$ two and $\mathbf{b}$ five crystals. The black squares show the reference data with the air gap between crystals. Orange dots represent interference fringes with the injected $\mathrm{CO}_{2}$ gas. Black and orange lines show the fitting of the reference data and the data with injected $\mathrm{CO}_{2}$, respectively. The coefficient of determination $\left(R^{2}\right)$ for the two- and five-crystal interferometers is $\sim 0.88$ and 0.82 , respectively. The vertical scale shows the CCD counts.

and five crystals, respectively. The reference measurement is taken with air between the crystals. The points correspond to the experimental data; the solid lines show the fitting of the experimental data using Eq. (6).

From the fitting of the experimental data, we find that the relative shift of the interference fringes for the twocrystal interferometer is $\Delta \phi_{2}=-(0.167 \pm 0.015) \pi$, and in the five-crystal interferometer, it is $\Delta \phi_{5}=-(0.187 \pm$ $0.009) \pi$. Thus, the precision in the measurement of the phase shift in the five-crystal interferometer is 1.66 times higher than that in the two-crystal interferometer. This value is consistent with the reduction in the width of the bright interference fringe; see Fig. 4 . The sensitivity of $\mathrm{CO}_{2}$ detection in the current five-crystal configuration is $1.8 \times 10^{3} \mathrm{ppm}$.

The sensitivity of this method can be further improved by using more nonlinear crystals and increasing the interaction length. In practice, such a device can be realized using a high-finesse cavity with just a single crystal. We estimate that for a cavity with a finesse of 150 and a base of $80 \mathrm{~mm}$, the theoretical value of the sensitivity reaches a level of a few tens of ppm, which is comparable with that of compact commercial optical sensors ${ }^{40}$.

\section{An interferometer with a "defect" in the superlattice}

Our experimental setup allows full flexibility in investigating nonlinear interferometers with variable crystal configurations. To demonstrate this, we remove the third crystal from the interferometer and observe the interference from the first, second, fourth, and fifth crystals (assuming air between the crystals). We use Eq. (5) to calculate the interference pattern, which in this case is given by:

$$
I \propto\left\{\operatorname{sinc}\left(\frac{\Delta k l}{2}\right) \cdot \cos \left[\Delta k l+\frac{3 \Delta k^{\prime} l^{\prime}}{2}+\frac{\Delta k^{\prime} l}{2}\right] \cos \left[\frac{\Delta k l}{2}+\frac{\Delta k^{\prime} l^{\prime}}{2}\right]\right\}^{2}
$$

The theoretical interference pattern given by Eq. (8) is shown in Fig. 7a, and the corresponding experimental results are shown in Fig. 7b. The results are found to be in good agreement. As one can see, the interference pattern contains additional contributions originating from 

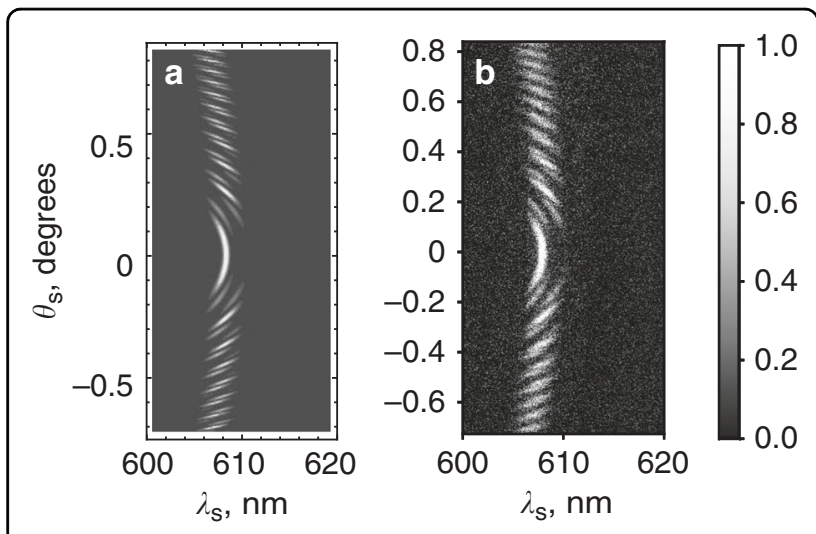

Fig. 7 Interference in the superlattice with a "defect". Normalized frequency-angular spectra (interference patterns) in the case when the third crystal is removed from the interferometer. The interference from the first, second, fourth, and fifth crystals is observed: a theoretical and b experimental dependencies. The parameters of the interferometer are similar to those in Figs. 6 and 7 with an air gap between the crystals. The data are normalized to the maximum counts of the CCD camera.

interferometers with different gaps. The ability to manipulate the interference patterns opens up possibilities for quantum state engineering ${ }^{20,35}$.

\section{Discussion}

We realized a nonlinear interferometer with a crystal superlattice with up to five nonlinear elements. We experimentally demonstrated that with an increase in the number of nonlinear elements, the interference fringes become narrower, which directly translates to improved sensitivity in metrological and sensing applications. The observed effect originates from the constructive interference of wavefunctions of down-converted photons, which are coherently generated in different crystals. The effect is clearly analogous with classic multi-source interference. We also found that an interferometer with a crystal superlattice becomes increasingly dependent on the accuracy in setting the experimental parameters, in particular, the phase-matching angles of the crystals. This reflects the common property of multiple-beam interferometers, which are more demanding for the settings of individual elements.

The presented configuration allows flexibility in the realization of unconventional crystal configurations, for example, by setting different gaps between crystals and using crystals of different sizes, which opens an interesting possibility for quantum state engineering.

We anticipate that our work will trigger more than one creative design in the realization of complex nonlinear interferometers with correlated photons, such as by using mirrors, integrated photonics, or fibre platforms. It is also of interest to investigate this scheme for the high-gain regime of parametric down-conversion ${ }^{41}$. We believe that the presented concept will provide a viable path towards high-performance devices for sensing, metrology, and quantum state engineering. When finalizing this work, we became aware of relevant work on the realization of a three-stage nonlinear interferometer ${ }^{42}$.

\section{Materials and methods}

Our experimental setup is shown in Fig. 1b. A continuous-wave laser $(\mathrm{cw})$ with a wavelength of $532 \mathrm{~nm}$ (60 mW, Laser Quantum) pumps a set of identical lithium niobate nonlinear crystals cut from a single master crystal (5\% $\mathrm{MgO}_{\mathrm{LiNbO}}, l=1 \mathrm{~mm}$, cut angle of $48.5^{\circ}$, Eksma Optics). Each crystal is coated with broadband AR coating, which introduces less than $1 \%$ loss for pump and signal photons and less than $5 \%$ loss for idler photons. The crystals are separated by the distance $l^{\prime}=8.2 \mathrm{~mm}$. They are mounted on a kinematic prism mount (KM100PM Thorlabs) and clamped by a small adjustable clamping arm (PM3, Thorlabs). The mount provides $0.45 \mathrm{deg}$ adjustment per revolution. The estimated experimental accuracy in setting the phase-matching angle of the crystal is $\Delta \theta_{c}= \pm 0.02^{\circ}$.

Photon pairs are generated in each nonlinear crystal in the type-I quasi-collinear frequency nondegenerate regime. A notch filter NF and a polarizer $\mathrm{V}$ are used to filter out the pump. Signal photons are focused on the slit of the imaging spectrograph (Acton) using the lens $\mathrm{F}(f=$ $300 \mathrm{~mm}$ ). The interference pattern of signal photons in frequency-angular coordinates is recorded by a CCD camera for visible light (Andor iXon 897) at the output of the spectrometer. The camera has $512 \times 512$ pixels and a pixel size of $16 \mu \mathrm{m}$, the gain of the camera is set to 290 , and the temperature of the camera sensor is kept at $-80^{\circ} \mathrm{C}$. The optical noise is measured independently and taken into account at the stage of data processing.

To ensure the indistinguishability of photon pairs produced in every crystal of the superlattice, all the SPDC photons should be generated and propagate within the interaction volume defined by the pump beam. This requirement is expressed in the condition $\left(2 l+l^{\prime}\right) \tan$ $\left(\theta_{\mathrm{s}}\right) \ll d$, which links the scattering angle $\theta_{\mathrm{s}}$, pump diameter $d$, and parameters of the superlattice $l, l^{\prime}$ (ref. ${ }^{16,26}$ ). To satisfy this condition, we set $d \sim 3 \mathrm{~mm}$ using the beam expander (LS) and detect angles up to $\theta_{\mathrm{s}}= \pm 0.85^{\circ}$.

Obtaining interference patterns with high visibility requires careful alignment of the interferometer. First, the orientation of each crystal is set to generate identical frequency spectra, which are measured by the spectrograph; see Section 5 of the Supplementary Materials. Then, by observing pairwise interference fringes between crystals, we ensure that the optical axes of the crystals are aligned in the same direction. Next, the distances between the crystals are carefully aligned to ensure equal gaps 
between them; see Sections 3 and 6 of the Supplementary Materials. Each crystal is mounted on a 2D translation stage so that it can be moved in and out of the interferometer. By successively observing the interference patterns from two, three, and four crystals, we adjust the distances between the crystals such that the fringes overlap. The accuracy of setting the length of the gap $l$ ' between crystals by this method is better than $100 \mu \mathrm{m}$. After the alignment of the crystals, we perform measurements of the interference with different numbers of crystals in the superlattice. The influence of the crystal length and the gap between the crystals on the interference pattern is analysed in detail in Section 7 of the Supplementary Materials.

In the gas sensing experiments, the interferometer is placed in an airtight enclosure (marked by a dashed rectangle) with an input socket for carbon dioxide gas $\left(\mathrm{CO}_{2}\right.$, 99.9\% purity). The wavelength of the idler photons is set to match the absorption peak at $\sim 4.27 \mu \mathrm{m}$. The gas homogeneously fills the volume between the crystals. Its concentration in the enclosure is controlled by a commercial $\mathrm{CO}_{2}$ sensor (Amphenol, accuracy $\pm 10 \%$ of reading). The experiments are conducted at a room temperature of $22^{\circ} \mathrm{C}$.

\section{Acknowledgements}

We acknowledge the support of the Quantum Technology for Engineering (QTE) programme of A*STAR and of the NRF CRP grant NRF-CRP14-2014-04. We are grateful to Sergei Kulik, Radim Fillip, Maria Chekhova, Galiya Kitaeva, and Berthold-Georg Englert for stimulating discussions.

\section{Author contributions}

L.A.K. conceived the idea of the experiments and supervised the project. A.V.P. built the experimental setup, performed measurements, and conducted numerical simulations. All the authors discussed the results and contributed to writing the manuscript.

\section{Conflict of interest}

The authors declare that they have no conflict of interest.

Supplementary information is available for this paper at https://doi.org/ 10.1038/s41377-020-0320-1.

Received: 3 November 2019 Revised: 8 April 2020 Accepted: 21 April 2020 Published online: 09 May 2020

\section{References}

1. Taylor, M. A. \& Bowen, W. P. Quantum metrology and its application in biology. Phys. Rep. 615, 1-59 (2016).

2. Dorfman, K. E., Schlawin, F. \& Mukamel, S. Nonlinear optical signals and spectroscopy with quantum light. Rev. Mod. Phys. 88, 045008 (2016).

3. Phan, N. M. et al. Interaction of fixed number of photons with retinal rod cells. Phys. Rev. Lett. 112, 213601 (2014).

4. Schlawin, F., Dorfman, K. E. \& Mukamel, S. Entangled two-photon absorption spectroscopy. Acc. Chem. Res. 51, 2207-2214 (2018).

5. Nasr, M. B. et al. Demonstration of dispersion-canceled quantum-optical coherence tomography. Phys. Rev. Lett. 91, 083601 (2003).

6. Okano, M. et al. $0.54 \mu \mathrm{m}$ resolution two-photon interference with dispersion cancellation for quantum optical coherence tomography. Sci. Rep. 5, 18042 (2015).

7. Graciano, P. Y. et al. Interference effects in quantum-optical coherence tomography using spectrally engineered photon pairs. Sci. Rep. 9, 8954 (2019).
8. Ono, T., Okamoto, R. \& Takeuchi, S. An entanglement-enhanced microscope. Nat. Commun. 4, 2426 (2013).

9. Taylor, M. A. et al. Biological measurement beyond the quantum limit. Nat. Photonics 7, 229-233 (2013)

10. Kalashnikov, D. A. et al. Quantum spectroscopy of Plasmonic nanostructures. Phys. Rev. X 4, 011049 (2014).

11. Zou, X. Y., Wang, L. J. \& Mandel, L. Induced coherence and indistinguishability in optical interference. Phys. Rev. Lett. 67, 318-321 (1991).

12. Wang, L. J., Zou, X. Y. \& Mandel, L. Induced coherence without induced emission. Phys. Rev. A 44, 4614-4622 (1991).

13. Yurke, B., McCall, S. L. \& Klauder, J. R. SU(2) and SU $(1,1)$ interferometers. Phys. Rev. A 33, 4033-4054 (1986).

14. Herzog, T. J. et al. Frustrated two-photon creation via interference. Phys. Rev Lett. 72, 629-632 (1994).

15. Chekhova, M. V. \& Ou, Z. Y. Nonlinear interferometers in quantum optics. Adv. Opt. Photonics 8, 104-155 (2016).

16. Kulik, S. P. et al. Two-photon interference in the presence of absorption. J. Exp. Theor. Phys. 98, 31-38 (2004)

17. Kitaeva, G. K. et al. A method of calibration of terahertz wave brightness under nonlinear-optical detection. J. Infrared Millim. Terahertz Waves 32, 1144 (2011).

18. Manceau, M. et al. Detection loss tolerant supersensitive phase measurement with an SU $(1,1)$ interferometer. Phys. Rev. Lett. 119, 223604 (2017).

19. Hudelist, F. et al. Quantum metrology with parametric amplifier-based photon correlation interferometers. Nat. Commun. 5, 3049 (2014).

20. Riazi, A. et al. Biphoton shaping with cascaded entangled-photon sources. npj Quantum Inf. 5, 77 (2019).

21. Liu, Y. H. et al. Loss-tolerant quantum dense metrology with $S U(1,1)$ interferometer. Opt. Express 26, 27705-27715 (2018).

22. Ono, T. et al. Observation of nonlinear interference on a silicon photonic chip. Opt. Lett. 44, 1277 (2019).

23. Solntsev, A. S. et al. LiNbO3 waveguides for integrated SPDC spectroscopy. APL Photonics 3, 021301 (2018).

24. Lemos, G. B. et al. Quantum imaging with undetected photons. Nature $\mathbf{5 1 2}$, 409-412 (2014).

25. Korystov, D. Y., Kulik, S. P. \& Penin, A. N. Rozhdestvenski hooks in two-photon parametric light scattering. J. Exp. Theor. Phys. Lett. 73, 214-218 (2001).

26. Kalashnikov, D. A. et al. Infrared spectroscopy with visible light. Nat. Photonics 10, 98-101 (2016)

27. Paterova, A. V. et al. Measurement of infrared optical constants with visible photons. N. J. Phys. 20, 043015 (2018).

28. Vallés, A. et al. Optical sectioning in induced coherence tomography with frequency-entangled photons. Phys. Rev. A 97, 023824 (2018).

29. Paterova, A. V. et al. Tunable optical coherence tomography in the infrared range using visible photons. Quantum Sci. Technol. 3, 025008 (2018).

30. Paterova, A. V. et al. Polarization effects in nonlinear interference of downconverted photons. Opt. Express 27, 2589-2603 (2019).

31. Klyshko, D. N. Ramsey interference in two-photon parametric scattering. J. Exp. Theor. Phys. 104, 2676-2684 (1993).

32. U'Ren, A. B. et al. Generation of two-photon states with an arbitrary degree of entanglement via nonlinear crystal superlattices. Phys. Rev. Lett. 97, 223602 (2006).

33. Lee, S. K, Yoon, T. H. \& Cho, M. Quantum optical measurements with undetected photons through vacuum field indistinguishability. Sci. Rep. 7, 6558 (2017).

34. Belinsky, A. V. \& Klyshko, D. N. Two-photon wave packets. Laser Phys. 4, 663-689 (1994).

35. $\mathrm{Su}$, J. et al. Versatile and precise quantum state engineering by using nonlinear interferometers. Opt. Express 27, 20479-20492 (2019).

36. Burlakov, A. V. et al. Interference effects in spontaneous two-photon parametric scattering from two macroscopic regions. Phys. Rev. A 56, 3214-3225 (1997).

37. Korystov, D. Y., Kulik, S. P. \& Penin, A. N. Interferometry of spontaneous parametric light scattering. Quantum Electron. 30, 922-926 (2000).

38. Klyshko, D. N. Parametric generation of two-photon light in anisotropic layered media. J. Exp. Theor. Phys. 105, 1574-1582 (1994).

39. Born, M. \& Wolf, E. Principles of Optics. (Cambridge University Press, Cambridge, 1999).

40. Yasuda, T., Yonemura, S. \& Tani, A. Comparison of the characteristics of smal commercial NDIR CO2 sensor models and development of a portable CO2 measurement device. Sensors 12, 3641-3655 (2012).

41. Kolobov, M. I. et al. Controlling induced coherence for quantum imaging. J. Opt. 19, 054003 (2017).

42. Li, J. M. et al. Generation of pure-state single photons with high heralding efficiency by using a three-stage nonlinear interferometer. https:/arxiv.org/ abs/2002.00314 (2020). 